\title{
The clinical benefit of array-based comparative genomic hybridization for detection of copy number variants in Czech children with intellectual disability and developmental delay
}

\author{
Marketa Wayhelova ${ }^{1,2^{*}}$, Jan Smetana ${ }^{1,2}$, Vladimira Vallova ${ }^{1,2}$, Eva Hladilkova ${ }^{2}$, Hana Filkova ${ }^{2}$, Marta Hanakova ${ }^{2}$,
} Marcela Vilemova ${ }^{2}$, Petra Nikolova ${ }^{2}$, Barbora Gromesova ${ }^{2}$, Renata Gaillyova ${ }^{2}$ and Petr Kuglik ${ }^{1,2}$

\begin{abstract}
Background: Chromosomal microarray analysis has been shown to be a valuable and cost effective assay for elucidating copy number variants (CNVs) in children with intellectual disability and developmental delay (ID/DD).

Methods: In our study, we performed array-based comparative genomic hybridization (array-CGH) analysis using oligonucleotide-based platforms in 542 Czech patients with ID/DD, autism spectrum disorders and multiple congenital abnormalities. Prior to the array-CGH analysis, all the patients were first examined karyotypically using Gbanding. The presence of CNVs and their putative derivation was confirmed using fluorescence in situ hybridization (FISH), multiplex ligation-dependent probe amplification (MLPA) and predominantly relative quantitative polymerase chain reaction ( $\mathrm{QPCR}$ ).
\end{abstract}

Results: In total, 5.9\% (32/542) patients were positive for karyotypic abnormalities. Pathogenic/likely pathogenic CNVs were identified in $17.7 \%$ of them (96/542), variants of uncertain significance (VOUS) were detected in $4.8 \%$ (26/542) and likely benign CNVs in 9.2\% of cases (50/542). We identified 6.6\% (36/542) patients with known recurrent microdeletion (24 cases) and microduplication (12 cases) syndromes, as well as 4.8\% (26/542) patients with non-recurrent rare microdeletions (21 cases) and microduplications (5 cases). In the group of patients with submicroscopic pathogenic/ likely pathogenic CNVs (13.3\%; 68/510) we identified 91.2\% (62/68) patients with one CNV, 5.9\% (4/68) patients with two likely independent CNVs and 2.9\% (2/68) patients with two CNVs resulting from cryptic unbalanced translocations. Of all detected CNVs, 21\% (31/147) had a de novo origin, 51\% (75/147) were inherited and 28\% (41/147) of unknown origin.

In our cohort pathogenic/likely pathogenic microdeletions were more frequent than microduplications (69\%; 51/74 vs. $31 \%$; 23/74) ranging in size from $0.395 \mathrm{Mb}$ to $10.676 \mathrm{Mb}$ (microdeletions) and $0.544 \mathrm{Mb}$ to $8.156 \mathrm{Mb}$ (microduplications), but their sizes were not significantly different $(P=0.83$ ). The pathogenic/likely pathogenic CNVs (median $2.663 \mathrm{Mb}$ ) were significantly larger than benign CNVs (median $0.394 \mathrm{Mb})(P<0.00001)$ and likewise the pathogenic/likely pathogenic CNVs (median $2.663 \mathrm{Mb}$ ) were significantly larger in size than VOUS (median $0.469 \mathrm{Mb})(P<0.00001)$.

* Correspondence: marketa.wayhelova@mail.muni.cz

${ }^{1}$ Institute of Experimental Biology, Faculty of Science, Masaryk University, Kotlarska 267/2, Brno, Czech Republic

2Department of Medical Genetics, University Hospital Brno, Cernopolni 212/9,

Brno, Czech Republic

(c) The Author(s). 2019 Open Access This article is distributed under the terms of the Creative Commons Attribution 4.0 International License (http://creativecommons.org/licenses/by/4.0/), which permits unrestricted use, distribution, and reproduction in any medium, provided you give appropriate credit to the original author(s) and the source, provide a link to the Creative Commons license, and indicate if changes were made. The Creative Commons Public Domain Dedication waiver (http://creativecommons.org/publicdomain/zero/1.0/) applies to the data made available in this article, unless otherwise stated. 
(Continued from previous page)

Conclusions: Our results confirm the benefit of array-CGH in the current clinical genetic diagnostics leading to identification of the genetic cause of ID/DD in affected children.

Keywords: Intellectual disability, Developmental delay, Microdeletion, Microduplication, CNV, Array-CGH

\section{Background}

Intellectual disability and developmental delay (ID/DD) represent a worldwide health and social problem with a prevalence of $1-3 \%$ in children from Western European population. These patients are characterized by significantly reduced abilities to understand new or complex information and to learn and apply new skills, resulting in a limited ability to cope independently. The deficiencies manifest before the 18th year, with lasting effect on development [1]. Most children with mild ID are diagnosed in early school age because of developmental delay and developmental disorders, while children with severe forms of ID can receive their clinical diagnose earlier, within the first 2 years of life [2]. ID/DD can occur as part of clinically defined rare syndromes in combination with multiple congenital anomalies (MCA) or other neurological and neurodevelopmental features as epilepsy, sensory disorders or autism spectrum disorders (ASD) with highly variable severity (syndromic ID/ $\mathrm{DD}$ ), while non-syndromic ID/DD patients typically manifest ID/DD as a single clinical feature [3]. In clinical practice, these categories often merge due to less apparent dysmorphic or other abnormal features which may be overlooked. The genetic background of ID/DD and ASD is recognised as the most common identifiable cause in their pathogenic progression. Recent worldwide studies frequently demonstrate extreme genetic heterogeneity in the pathogenesis of ID/DD and ASD varying from large chromosomal abnormalities to point mutations in single genes that have been documented in a wide spectrum of copy-number variants $(\mathrm{CNVs})$, single gene sequence variants or small insertions, deletions or duplications in DNA sequence or defects in the epigenetic regulation of gene expression [3-5].

In current genetic diagnostics, the method of arraybased comparative genomic hybridization (array-CGH) has become a routine analytical technique for the detection of CNVs in patients with ID/DD, ASD and MCA. In general, there are two major groups of CNVs: recurrent and non-recurrent CNVs. Briefly, recurrent CNVs arise following non-allelic homologous recombination (NAHR) during meiosis between low-copy repeats encompassing large gene-rich blocks as reviewed by Weise et al. (2012) and Watson et al. (2014) [6, 7]. This model is evidenced by the occurrence of breakpoints in the same or a very similar genomic position (allowing for differences between the resolutions of microarray platforms) in patients with recurrent microdeletions/ microduplications. Conversely, non-recurrent unique CNVs arise from different events that do not depend directly on genomic architecture, and include non-homologous end joining (NHEJ) and processes of fork stalling and template switching (FoSTeS) [8-10]. Recent studies on large cohorts show that submicroscopic chromosomal rearrangements can explain the pathological phenotype in about 15-20\% children with ID/DD $[11,12]$. Due to its high sensitivity, accuracy and diagnostic yield, array-CGH is now recommended as a first-tier test for ID/DD [12, 13]. In the past, array-CGH led to identification of multiple microdeletion/ microduplication syndromes and still facilitates the detection and characterization of novel, cryptic submicroscopic, non-recurrent chromosomal rearrangements. Although "next-generation" sequencing (NGS) is now rapidly evolving as the ultimate approach to molecular genetic diagnostics of children with ID/DD, array-CGH still represents widespread gold standard in the diagnostic practice.

In this study we report the diagnostic yield of pathogenic/likely pathogenic recurrent and non-recurrent CNVs on a cohort of 542 Czech children with ID/DD, ASD and MCA. Simultaneously we demonstrate the usefulness of array-CGH as a clinical test in the genetic counselling and evolving challenges in the data interpretation.

\section{Methods}

A total of 542 children with ID/DD, ASD and MCA were diagnosed at the Department of Medical Genetics (University Hospital Brno, Czech Republic) in the period from 2012 to 2017, including 215 girls and 327 boys with median age of 5 years ( $0-18$ years). Almost $60 \%$ $(315 / 542)$ were examined before the age of six years $(0-$ 5 years) and only $5 \%(28 / 542)$ were older than 15 years (16-18 years) at the time of array-CGH analysis.

Prior to any genetic analysis being performed, parents signed an informed consent. All patients first received standard karyotyping using a G-banding procedure. The G-banded karyotypes were analysed, documented and archived for each patient using the LUCIA Cytogenetics ${ }^{\mathrm{Tm}}$ image analysis system (Laboratory Imaging, Prague, Czech Republic). Genomic DNA samples were obtained from leukocytes of peripheral blood in EDTA according to the standard DNA isolation process using MagNaPure system (Roche Ltd., Basel, Switzerland). Their quality and quantity were assessed by NanoDrop ${ }^{\circ}$ ND-1000 
(ThermoFisher Scientific, Waltham, MA, USA) and Qubit $^{\circ} 1.0$ (ThermoFisher Scientific).

The whole-genomic screening of chromosomal rearrangements by array-CGH was performed using DNA microarray platforms (180 K and $60 \mathrm{~K})$ : SurePrint G3 Human CGH + SNP Microarray 4x180K, SurePrint G3 Human CGH Microarray 4x180K, SurePrint G3 Human CGH Microarray 8x60K (Agilent Technologies, Santa Clara, CA, USA) and Cytosure ISCA 4X180K UPD array (Oxford Gene Technology, Oxfordshire, UK) according to the manufacturers' recommendations. The technical characteristics of all microarray platforms are available on manufacturer's websites. All samples were matched with Human Genomic DNA reference (Agilent Technologies or Promega, Madison, WI, USA). Microarray slides were scanned with a DNA Microarray Scanner (Agilent Technologies). Data were obtained using the Agilent Feature Extraction software 12.0.2.2 and Agilent CytoGenomics 4.0, respectively, and visually checked using the Agilent Genomic Workbench Software 7.0.4.0 and Agilent CytoGenomics 4.0. CNVs were detected using the ADM-2 algorithm with filters of minimal size of $200 \mathrm{~kb}$ in region, $>5 \mathrm{Mb}$ of copy-number neutral loss of heterozygosity $(\mathrm{cnnLOH})$ regions (in $\mathrm{CGH}+\mathrm{SNP}$ analyses), and a minimal absolute average log ratio of 0.25 as cut-off. Genomic positions were estimated using the human genomic reference sequence GRCh37/hg19 when using the DNA microarray platforms of Agilent Technologies, and the reference sequence GRCh36/hg18 when employing DNA microarray platforms by Oxford Gene Technology (OGT).

\section{CNV analysis and classification}

For clinical use, every detected non-polymorphic CNV requires interpretation. As outlined elsewhere [14, 15], we considered a set of minimal criteria to interpret $\mathrm{CNVs}$ as 1) pathogenic or likely pathogenic CNVs, 2) variants of uncertain significance (VOUS), 3) likely benign CNVs and 4) variants with insufficient evidence for their clinical significance (not assessed). We followed the decision tree as presented by Buysse et al. (2009) [16]. The two-tailed Mann-Whitney U test was used to compare CNVs sizes, the test was run at the $5 \%$ level of significance.

\section{CNV confirmatory testing FISH and MLPA analysis}

Fluorescence in situ hybridization (FISH) and multiplex ligation-dependent probe amplification (MLPA) were used to confirm the CNV findings and establish the inheritance, as described elsewhere [17-19]. The confirmatory method was selected according to availability of parental genetic material and perceived clinical value of the given method.

\section{Relative quantitative $P C R$ ( $q P C R$ )}

Relative qPCR was performed with two pairs of DNA primers which were designed to prime both the inside and outside of CNVs, and one pair of DNA primers targeting the $E R H$ gene as an endogenous control. The reactions were run in duplicates, following the manufacturer's recommendations (ThermoFisher Scientific). $C_{\mathrm{T}}$ values were subtracted for the $E R H$ gene and each tested DNA region, for which was evaluated to derive R-values. An R-value < 0.7 for DNA loss and $>1.3$ for DNA gain in the targeted DNA regions relatively to the $E R H$ gene were set as cutoffs. The DNA primers were synthesized using a commercially available service (Integrated DNA Technologies, Coralville, Iowa, USA).

\section{Results}

In the 6-year period from 2012 to 2017, a total of 542 children with ID/DD, MCA and ASD were examined according to afore mentioned multi-step molecular genetic diagnostic procedure at the Department of Medical Genetics (University Hospital Brno). First their karyotype was examined by G-banding followed by whole-genomic screening of $\mathrm{CNVs}$ by microarray. Using array-CGH, $32.7 \%$ of them (177/542) exhibited non-polymorphic $\mathrm{CNVs}$ or large regions of cnnLOH.

Overall, we identified clinically relevant CNVs for ID/ DD and associated comorbidities in $17.7 \%$ of patients (96/542) which were classified as pathogenic or likely pathogenic CNVs, the VOUS were detected in $4.8 \%$ of cases (26/542) and benign CNVs in $9.2 \%$ of cases (50/ 542). Among all 542 cases, $5.9 \%$ patients had initial cytogenetic abnormalities (32/542) which were subsequently confirmed and specified by array-CGH $(100 \%$; $32 / 32)$ (see Additional file 1).

An example of a positive cytogenetic finding in karyotype that was subsequently confirmed by array-CGH (patient 280) is presented in the Fig. 1a, b.

The 2-year-old male patient with developmental delay and congenital malformations of his genital organs had: a) the karyotype analysis identified an unbalanced translocation $t(X ; Y)$ and the presence of normal $Y$ chromosome. The derived chromosome $\mathrm{X}$ was inherited from an affected mother with a karyotype 46,X,t $(\mathrm{X} ; \mathrm{Y})(\mathrm{p} 22.3 ; \mathrm{q} 11.21)$.

b) The array-CGH analysis identified a Xp22.31p22.32 microdeletion (5.83 Mb) and Yq11.221-q12 duplication $(43.01 \mathrm{Mb})$. The patient has no copy of Xp22.31p22.32 locus and two copies of Yq11.221-q12 locus. Combining both karyotype and array-CGH analyses, the Yq11.221-q12 part is translocated to the short arm of X chromosome lacking Xp22.31-p22.32 locus.

\section{The findings of single pathogenic/likely pathogenic CNV} Considering 510 patients with normal karyotype, we detected submicroscopic pathogenic or likely pathogenic 

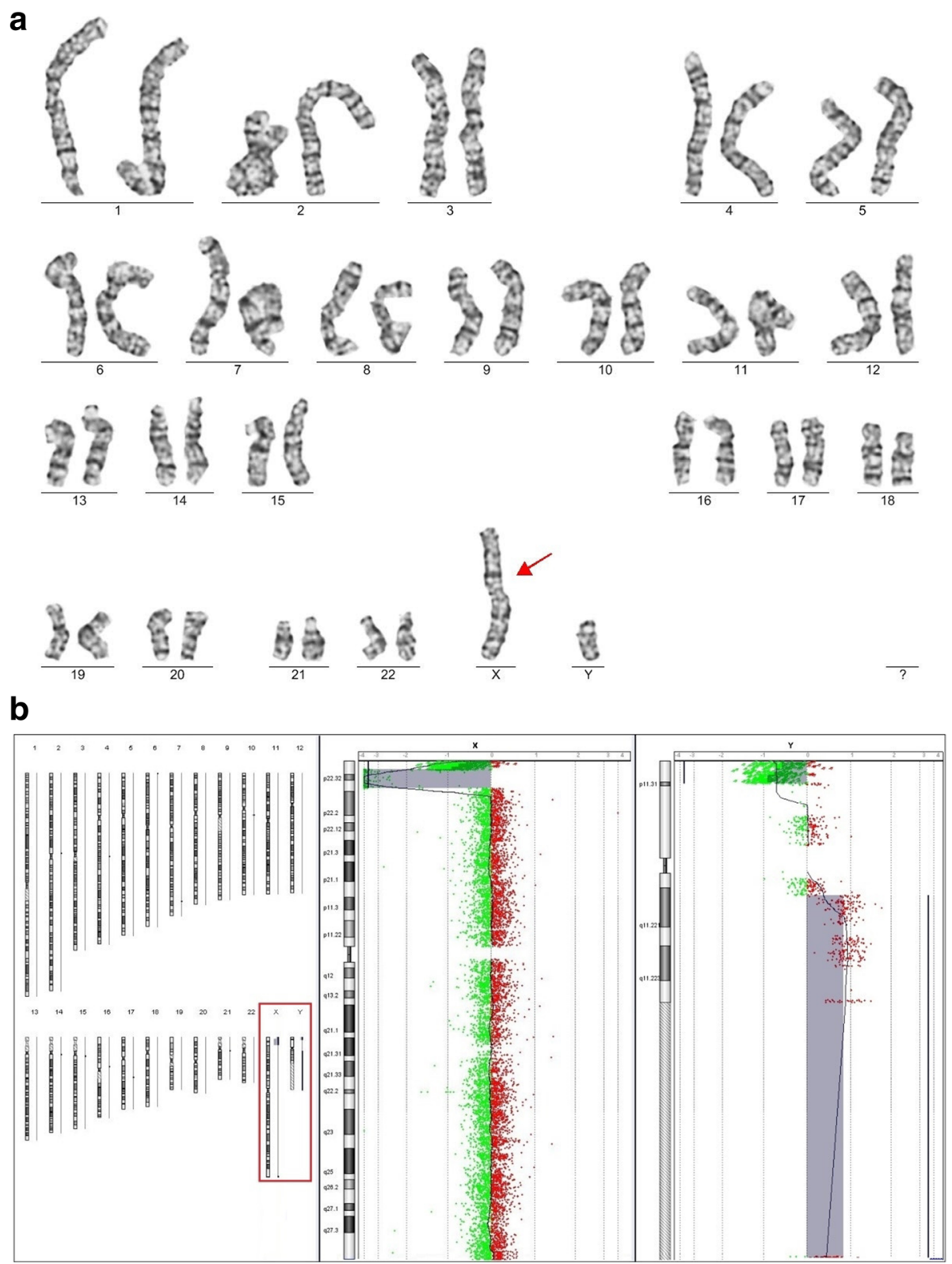

Fig. 1 The example of a patient with the cytogenetic finding in the karyotype and array-CGH profile with specification of this chromosomal aberration. a The cytogenetic analysis of karyotype: $46, \operatorname{der}(X) t(X ; Y), Y$. The 2-year-old male patient with developmental delay and congenital malformations of genital organs has a karyotype $46, \operatorname{der}(X) t(X ; Y), Y$ and array-CGH has confirmed and specified this chromosomal aberration. $\mathbf{b}$ The array-CGH profile presenting array-CGH result: arr [GRCh37] Xp22.33p22.31(2701273_8531171)×0,Yq11.221q12(15991426_59002403)×2. The 2year-old male patient with developmental delay and congenital malformations of genital organs has a karyotype $46, \operatorname{der}(X) t(X ; Y), Y$ and array-CGH has confirmed and specified this chromosomal aberration

CNVs in $13.3 \%$ patients (68/510). In $12.2 \%(62 / 510)$ one clinically relevant CNV was identified. The group included 24 patients diagnosed with recurrent microdeletion syndromes: 1q21.1-q21.2 (3x), 1q43-q44 (3x), 7q11.23 (1x), 15q11.2 (3x), 15q1.1-q13.1 (1x), 15q13.2q13.3 (1x), 16p11.2 (3x), 16p12.2 (1x), 16p13.11 (1x), $17 \mathrm{p} 11.2(1 \mathrm{x}), 17 \mathrm{q} 12(1 \mathrm{x}), 22 \mathrm{q} 11.21$ or distal 22q11.21- q11.22 (4x) and 22q13.3 (1x). Also, 12 patients were diagnosed with recurrent microduplication syndromes: 1q21.1-q21.2 (2x), 15q11.2-q13.1 (3x), 16p11.2 (1x), $17 q 11.2(2 x), 17 q 12(1 x)$ and 22q11.21 (3x) (see Additional file 2). The remaining 26 cases were represented by non-recurrent microdeletion (21 cases) and microduplication CNVs (5 cases) (see Additional file 3). 


\section{The findings of two pathogenic/likely pathogenic CNVs (microdeletion/microduplication)}

In four cases the two likely independent pathogenic/likely pathogenic CNVs were detected. In patient 151, we identified a 2q12.1-q12.3 microduplication (3.411 Mb) and 2q13 microduplication (1.802 Mb). Parental DNA samples were not available to specify the origin of these CNVs. In the case of patient 274, we identified a 2q23.1-q23.3 microdeletion (4.922 Mb) and 7q21.12-q21.13 microduplication (1.682 Mb). In patient 21 , we identified maternally inherited $4 q 12-q 13.1 \quad(10.677 \mathrm{Mb})$ and $9 q 21.1 \quad(0.813 \mathrm{Mb})$ microdeletions with array-CGH. In the case of patient 451, we identified a de novo terminal 10q26.2-q26.3 microdeletion $(6.231 \mathrm{Mb})$ and maternally inherited interstitial 11p14.3-p15.1 microduplication (6.667 Mb).

Two other patients carried two pathogenic CNVs resulting from cryptic unbalanced translocations. In both cases, each patient was shown to carry one terminal microdeletion and one terminal microduplication indicating familial occurrence of a balanced translocation.

In the case of patient 380, we detected a terminal 7q36.1-q36.2 microdeletion $(6.827 \mathrm{Mb})$ and terminal 12p13.31-p13.33 microduplication (6.324 Mb): subtelomeric metaphase FISH showed the cryptic balanced translocation $t(7 ; 12)$ (q36.3;p13.33) to be of paternal origin.

Patient 430 was a female with a 46,XY karyotype. Using array-CGH we further detected a terminal 9p23-p24.3 microdeletion $(9.832 \mathrm{Mb})$ and terminal 16q23.3-q24.3 microduplication $(8.156 \mathrm{Mb})$ in which the size of both $\mathrm{CNVs}$ was at the resolution limit of classical cytogenetics. To date, the origin of these CNVs has not been assessed. The summaries of patients with two pathogenic/likely pathogenic CNVs are presented in Additional file 4.

\section{Classification and characteristics of CNVs}

Considering only the cases with pathogenic or likely pathogenic submicroscopic chromosomal rearrangements, microdeletions were more frequent than microduplications (69\%; 51/74 vs. 31\%; 23/74), however, their sizes (median 2.547 Mb for microdeletions, range 0.395-10.676 $\mathrm{Mb}$; median $2.823 \mathrm{Mb}$ for microduplications, range $0.544-8.156 \mathrm{Mb})$ did not differ significantly $(P=0.83)$.

Also, $4.8 \%$ of all array-CGH findings (26/542) were classified as VOUS. In detail, we identified total of 17 structural CNVs with uncertain significance in 15 patients, 3 cases presented only gonosomal aneuploidies (47,XXX in two cases and 47, XYY in one case) and 8 cases with large regions of $\mathrm{cnnLOH}>5 \mathrm{Mb}$. These outputs are presented in the Additional file 5. In general, the structural VOUS were typically gene-poor: 7 VOUS did not contain any disease-related OMIM genes, 6 VOUS contained per 1 disease-related gene, 1 VOUS contained 2 disease-related genes and 2 VOUS contained per 5 disease-related genes. Focusing on genotype-phenotype correlations the losses or gains of these genes did not fully explain the phenotypic abnormalities in our patients. In one case we detected maternally derived marker chromosome 21 (6.032 Mb), containing 2 disease-related OMIM genes. The size of remaining 16 submicroscopic VOUS varied from 0.24 $\mathrm{Mb}$ to $3.238 \mathrm{Mb}$ and microduplications were 3-fold frequent than microdeletions (12 vs. 4).

Using CGH + SNP array platform we detected 8 cases of large cnnLOH regions. In 6 cases the children were from consanguineous families so we observed more than one cnnLOH locus across their genomes. The remaining cases did not have a family history of consanguinity. These results providing some important information on the genomic location, size and gene content were essential for additional molecular genetic analyses by Sanger sequencing e.g. in patient 10 from consanguineous family. We identified cnnLOH $>5 \mathrm{Mb}$ in chromosomal loci 4q28.3, 10q11.22q21.2, 11q22.1q22.3 and 12q24.23q24.32 and analysed their gene content (see Additional file 5). Based on her phenotypic features of suspected Cockayne syndrome we performed Sanger sequencing of the ERCC6 gene which is located in the chromosomal locus 10q11.23. We detected a nonsense sequence variant c.3259C $>\mathrm{T}$ (p.Arg1087X) in homozygous state and confirmed the diagnosis on molecular genetic level (data in the medical record).

The CNVs in $9.2 \%$ cases were classified as likely benign with $57 \mathrm{CNVs}$ in 50 patients (50/542). To summarize, the likely benign $\mathrm{CNVs}$ were gene-poor, only $29.8 \%$ of them $(17 / 57)$ contained any disease-related OMIM gene or genes (see Additional file 6). In those cases, the gene content did not correlate with the patient's phenotype and $88.2 \%(15 / 17)$ these CNVs were proved to be inherited from normal parent. The size of likely benign $\mathrm{CNVs}$ varied from $0.205 \mathrm{Mb}$ to $1.469 \mathrm{Mb}$ and microduplications were detected 2-fold more frequently than microdeletions (39 vs. 18). In $0.9 \%$ cases (5/542) the clinical impact of detected CNVs could not be assessed due to either absence of parental DNA samples for confirmatory analysis or relevant literature about CNVs in these chromosome locations.

Among the 68 patients with 74 pathogenic/likely pathogenic CNVs and normal karyotype, 36.5\% CNVs (27/74) were de novo in origin, $25.7 \%$ CNVs (19/74) were inherited from a healthy or mildly affected parent (14 CNVs of maternal origin and $5 \mathrm{CNVs}$ of paternal origin). In the remaining cases (28/74) at least one parental DNA sample was not available for analysis. On the other hand, $94.7 \%$ all the likely benign CNVs (54/57) were inherited and only one had de novo origin. Among 15 cases of VOUS (including the patient with marker chromosome derived from chromosome 21) we identified the de novo origin of $17.6 \%$ CNVs (3/17) and $23.5 \%$ cases (4/17) of 
inherited CNVs; in remaining CNVs their origin has not been assessed so far.

Comparing the size of CNVs to their clinical impact, it is clear that the likely benign CNVs are mostly smaller than $1 \mathrm{Mb}$. We detected 57 likely benign CNVs (median size $0.394 \mathrm{Mb}$, range $0.205-1.469 \mathrm{Mb}$ ) and only two of them were larger than $1 \mathrm{Mb}$, but smaller than $1.5 \mathrm{Mb}$. In cases presenting submicroscopic CNVs of uncertain significance (median size $0.503 \mathrm{Mb}$, range $0.240-3.238 \mathrm{Mb}$ ), $50 \%$ CNVs $(8 / 16)$ were smaller than $0.5 \mathrm{Mb}$, but we also detected $25 \%$ CNVs (4/16) larger than $1 \mathrm{Mb}$. In contrast among 74 pathogenic or likely pathogenic CNVs detected in 68 patients with normal karyotype we observed that $86 \% \mathrm{CNVs}(64 / 74)$ were larger than $1 \mathrm{Mb}$. Categorizing the CNVs as either microdeletions and microduplications, we can observe the different size distribution between microdeletion and microduplication VOUS as shown in Fig. 2a, b.

\section{Discussion}

The introduction of DNA microarrays has introduced a major advance in the discovery of new submicroscopic rearrangements in the pathogenesis of idiopathic ID/DD and associated congenital abnormalities. This was reflected by a rapidly increased diagnostic yield so it was with little surprise that array-CGH was adopted and recommended as a first-tier clinical diagnostic test for such patients $[12,13]$. The discovery of many new microdeletion and microduplication loci across the human genome represents a challenge to determine the pathogenicity and clinical impact introduced by these chromosome rearrangements.

In the course of our 6-year study, we examined 542 children with ID/DD, ASD and MCA by array-CGH using oligonucleotide DNA microarrays. In $32.7 \%$ of them (177/542), we detected non-polymorphic CNVs or large regions of $\mathrm{cnnLOH}$ with potential clinical relevance. Determining the clinical impact of CNVs is the critical point in array-CGH data interpretation. To this end, we considered their size, gene content, inheritance pattern and information from databases and relevant literature $[12,14]$. Overall, we identified the pathogenic/ likely pathogenic CNVs in $17.7 \%$ patients (96/542) which corresponds to the generally held diagnostic yield of 15$20 \%$ based on many multicenter studies [20-23]. Further, $5.9 \%$ of all patients had associated karyotypic findings (32/542). Based on multiple studies, classical Gbanded karyotyping can identify chromosomal aberrations in about $3-5 \%$ of children with ID/DD (excluding Down syndrome and other known chromosome syndromes) $[12,24]$. We have shown that array-CGH provides highly reliable confirmatory test specifying the size and gene content of chromosomal aberrations detected by postnatal G-banding karyotype analysis.
In the group of patients with normal karyotype we identified 7.1\% (36/510) patients with known recurrent microdeletion and microduplication syndromes and 5.1\% (26/ 510) patients with non-recurrent microdeletions and microduplications. Consequently, we divided the patients sharing recurrent CNVs into small groups. These groups of patients having these single recurrent pathogenic/likely pathogenic CNVs share some notable phenotypic features as ID/DD, ASD and facial stigmatization, however, some phenotypic features are patient-specific. This may indicate the partial impact of multiple loci acting across the human genome as modifiers, or, especially in the cases of microdeletion syndromes, the different expression of alleles present in the hemizygous state [25]. The occurrence of phenotypic modifiers can also explain cases of inherited microduplication syndromes, especially from a healthy parent. These modifiers could be specific for every family and probably lead to incomplete penetrance and variable expressivity of the genes included in the detected CNVs. For example, in our cohort of 36 patients with single recurrent pathogenic/likely pathogenic CNVs we identified three cases of 15q11.2 microdeletion of maternal origin, one case of 15q11.2-q13.1 duplication of paternal origin and one case of maternal origin, one case of 22q11.21 microduplication inherited from a mildly affected mother and one case of 22q11.21 microduplication of paternal origin. In these cases, we can expect the modifying impact of multiple loci across the human genome, which could explain the more severe phenotype in children than in their parents or the variability of phenotypes among children sharing the same CNVs.

Rosenfeld et al. (2013) performed Bayesian computational analysis, which arose from the CNVs frequencies in more than 22,000 controls and more than 48,000 postnatal cases of ID/DD, autism and MCA [26]. They calculated the penetrance for a large variety of CNVs, including all those considered in this article and estimated very low penetrances eg. $10,4 \%$ in the case of the 15q11.2 microdeletion. In our cohort, we identified three cases of maternally inherited 15q11.2 microdeletion and in all of them the mother was unaffected or had very mild phenotype. 15q11.2 microdeletion has been recently defined as one of the most common chromosomal abnormality in the pathogenesis of ASD [27]. In contrast we also identified three cases of proximal 16p11.2 microdeletion (two of them of de novo origin). For this $\mathrm{CNV}$ the penetrance was calculated as Rosenfeld et al. (2013) as $46.8 \%$, which is compatible with its powerful adverse impact on the phenotype [26]. In our study, it was not surprising that de novo CNVs appeared to have a higher general penetrance. The penetrance analysis also supports the role of other CNVs (proximal 1q21.1 microduplication, $15 \mathrm{q} 11.2$ microdeletion, 16p13.11 microdeletion, 16p12.1 microdeletion, distal 16p11.2 

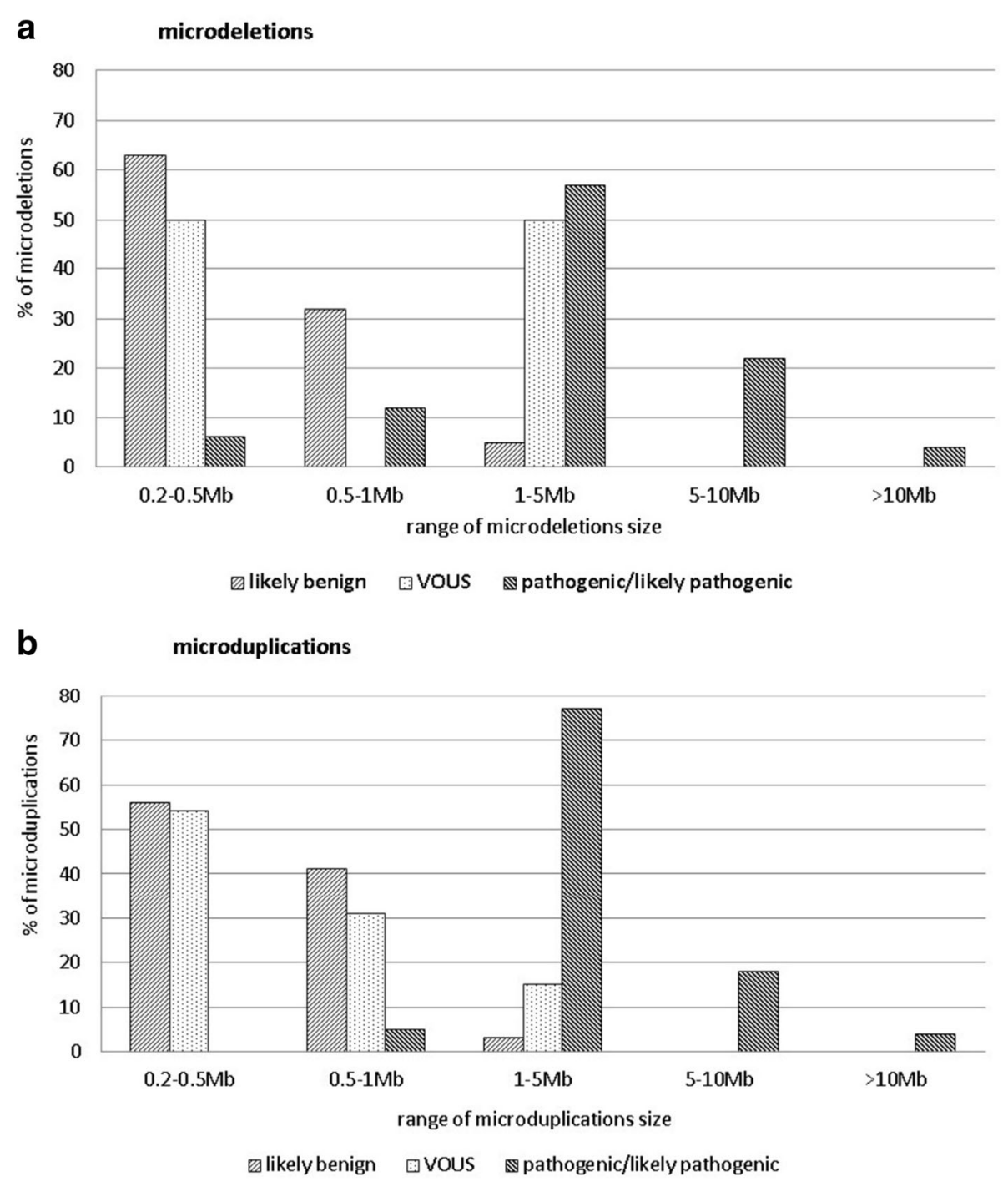

Fig. 2 The relationship between the size of submicroscopic CNVs and their clinical impact in children with ID/DD, ASD and MCA - a) microdeletions, b) microduplications. The data are based on the analysis of 147 CNVs detected in 132 children's patients with ID/DD, ASD and MCA. The CNVs observed in patients with positive cytogenetic findings and the cases with detected cnnLOH were excluded from this analysis. The sum of CNVs in every classification category is 100\%. a The relationship between the size of microdeletions and their clinical impact in children's patients with ID/DD, ASD and MCA. The data are based on the analysis of 147 CNVs detected in 132 children's patients with ID/DD, ASD and MCA. The CNVs observed in patients with positive cytogenetic findings in karyotype as well as those cases with detected LOH were excluded from this analysis. $\mathbf{b}$ The relationship between the size of microduplications and their clinical impact in children's patients with ID/DD, ASD and MCA. The data are based on the analysis of 147 CNVs detected in 132 children's patients with ID/DD, ASD and MCA. The CNVs observed in patients with positive cytogenetic findings in karyotype as well as those cases with detected LOH were excluded from this analysis

microdeletion) with low penetrance $(<20 \%)$ as "risk" or "susceptibility loci" in the pathogenesis of ID/DD, ASD and MCA, examples of which are present in our cohort.

Girirajan et al. (2010) presented a two-hit model for cases presenting severe neurodevelopmental delay [28]. They observed a significant proportion of cases with a second large $\mathrm{CNV}$ among cases with one specific $\mathrm{CNV}$ (16p12.1) compared to controls. They suggested the impact of "susceptibility loci" and the strong effect of a second $\mathrm{CNV}$ in the manifestation of severe phenotypic features. When the second CNV is not detected due to the resolution limit of microarray analysis, the approaches of whole-exome (WES) and whole-genome sequencing (WGS) can identify the second hit (rare or de novo DNA sequence variant or small $\mathrm{CNV}$ ) in cases with the CNVs acting as "risk" or "susceptibility loci" $[28,29]$.

In our cohort, we can clearly demonstrate the two-hit model in case 451 with a maternally inherited 11p14.3-p15.1 microduplication $(6.667 \mathrm{Mb})$ and de novo microdeletion 
10q26.2-q26.3 (6.231 Mb). The terminal 10q26.2-q26.3 microdeletion is already referred as the "Chromosome 10q26 deletion syndrome" (OMIM \#609625). Despite the presence of a 11p14.3-p15.1 microduplication (outside the BeckwithWiedemann critical region) the mother did not exhibit apparent phenotypic features, so we consider the presence of positively modifying effect of combined multiple loci across her genome and/or the absence of a second pathogenic hit as the most likely explanation.

Besides recurrent pathogenic/likely pathogenic CNVs, we detected a group of unique non-recurrent pathogenic/likely pathogenic CNVs. We analyzed them for their gene content to identify any candidate genes which could support their pathogenicity. Subsequently we estimated their origin in cases when parental DNA samples were available (see Additional file 3). As example, in patient 490 we detected 6q15-q161.1 microdeletion (2.141 $\mathrm{Mb}$ ) which affected the EPHA7 gene. Based on data from OMIM database (www.omim.org), this gene has not been clearly related to any congenital genetic disease so far. It encodes the ephrin type-A receptor of proteintyrosine kinase family. These receptors have been implicated in mediating development of the central nervous system [30]. Its role as a candidate gene in neurodevelopmental disorders is supported by a case of a 6q16.1 microdeletion in a child with neurological abnormalities [31].

In our cohort of patients with pathogenic/likely pathogenic submicroscopic CNVs we observed more than 2-fold higher frequency of microdeletions than microduplications (70\% vs. 30\%). Multiple studies proved the higher likehood of pathogenicity for microdeletions than for their reciprocal microduplications due to the content of dosage-sensitive haploinsufficient genes, then these microduplications usually cause milder phenotype [32, 33]. The level of pathogenicity of those CNVs can be also modified by the incomplete penetrance or by the position effect in the case of microduplications. In general, the vast majority of microduplications occur in tandem in orientation and are often inherited from healthy or slightly affected parent. However, the microduplication larger than $1 \mathrm{Mb}$ and located elsewhere in the genome, are expected to be likely pathogenic. For the clinical interpretation of microduplication it is generally recommended to assess their origin, to analyse the gene content and to search public databases of CNVs, and if possible, to locate them in the genome [34].

Concerning CNV size, we identified that CNVs larger than $1 \mathrm{Mb}$ are more likely to have pathogenic/likely pathogenic effect on phenotype. We also showed that vast majority of CNVs smaller than $500 \mathrm{~kb}$ had familial origin as reviewed in Miller et al. (2010) [12]. In detail, we detected a total of $46 \mathrm{CNVs}$ smaller than $500 \mathrm{~kb}$ with defined clinical impact: $76 \%$ of them had familial origin (35/46), $4 \%$ of them were de novo of origin $(2 / 46)$ and $20 \%$ of them $(9 / 46)$ have not assessed their origin so far.
Miller et al. (2010) also reviewed extensive studies based on HapMap samples, concluding that $90-95 \%$ of likely benign CNVs were smaller than $500 \mathrm{~kb}$. Based on the synthesis of multiple array-CGH analyses they recommend a resolution $\geq 400 \mathrm{~kb}$ for $\mathrm{CNVs}$ as a general analytical and clinical threshold. All these facts in data interpretation are necessary to evaluate every $\mathrm{CNV}$ independently in the context of the patient's phenotype, $\mathrm{CNV}$ origin, size, gene content and published information obtained from databases and literature. To demonstrate the CNV size as only one of more criteria to specify its clinical impact, we identified $58 \%$ of likely benign CNVs (33/57) smaller than $500 \mathrm{~kb}$, but on the other hand also three cases of known susceptibility regions for neurodevelopmental disorders (two cases of maternally derived 15q11.2 microdeletion and one case of 16p12.2 microdeletion of unknown origin) of size smaller than $500 \mathrm{~kb}$.

Using $180 \mathrm{~K} \mathrm{CGH}+$ SNP microarray platform we detected $1.5 \%$ cases $(8 / 542)$ with large stretches of cnnLOH $>5 \mathrm{Mb}$, in which we identified from tens to hundreds of genes related to autosomal recessive disorders. The presence of more than one $\mathrm{LOH}$ locus indicates the parental consanguinity which is typical for individuals from inbred populations [35, 36]. Based on low incidence of consanguinity in the Czech Republic ( 0.2\%) (www.consang.net) [37] we suggest to use CGH + SNP microarray platforms for analysis in specific families when the parental consanguinity is reported in the preliminary genetic counselling. As another step in molecular diagnostics algorithm, the approaches of targeted Sanger sequencing, WES or WGS can identify the particular pathogenic DNA sequence variants with autosomal recessive inheritance in these families [38, 39].

In the course of the study we used several types of DNA microarrays (180 K and $60 \mathrm{~K}$ ) but were unable to see any differences in their diagnostic reliability. So, although 180 $\mathrm{K}$ platforms offer a 3-fold higher resolution, we can also recommend the $60 \mathrm{~K}$ platforms for routine clinical arrayCGH analyses, as shown by Sansović et al. (2017) [23]. However, to confirm the complementarity and specificity of different microarray platforms we suggest reanalyses of DNA samples (with any detected CNVs) once or twice a year as an internal quality check for diagnostic laboratories. Based on the newly published recommendation, the microarray platform should achieve a resolution at least 200-400 kb for postnatal analyses, so both $60 \mathrm{~K}$ and $180 \mathrm{~K}$ microarray platforms are fully sufficient to comply this requirement $[12,39,40]$.

Following banding based karyotyping, array-CGH entered the field of molecular cytogenetic analyses and immediately significantly improved the diagnostic yield [41]. However, despite its high sensitivity, accuracy and reliability, arrayCGH cannot entirely replace the standard conventional G- 
banding karyotyping, due to its inability to detect balanced chromosomal rearrangements or to specify gains of particular DNA sequences in the karyotype. In some patients we proved the significance of the combined strategy including G-banding karyotyping and array-CGH. G-banding karyotyping facilities the global and complex analysis of chromosomal organization and array-CGH confirms and specifies the chromosomal rearrangements on molecular level.

Besides being a well-established routine test for the rapid detection of suspected microdeletion syndromes, FISH analysis can identify the position of duplicated loci in microduplication syndromes. Using specific locus probes, it is possible to identify cryptic balanced inversions in parents, resulting in CNVs arising in the offspring following meiotic recombination within the inversion segment of the carrier parent [42-44]. Thus, due to the wide range in its diagnostic capacity, array-CGH is a standard method in routine diagnostic multistep algorithm, where it serves as a first-tier test, confirmatory test or test following conventional G-banding karyotyping as well. Although our microarray studies have demonstrated the importance of de novo CNVs in ID/DD related disorders, the majority of our cases remain undiagnosed. Recent studies showed that WES/WGS can be applied for simultaneous detection and characterization of both CNVs and single-nucleotide variants, which could reduce the number of analyses to one complex test to reach the diagnosis [45]. In our pilot study using targeted NGS with commercially available gene panel we identified pathogenic sequence variants in some patients with negative array-CGH assay and confirm the diagnosis on the molecular level [46].

\section{Conclusions}

Our study confirms array-CGH as an effective diagnostic tool for both detection and precise characterization of clinically important CNVs in children with ID/DD, ASD and MCA. Due to its wide application and clear cost effectiveness, array-CGH now offers the most efficient cytogenetic screening method routinely used in clinical laboratories and it is only likely to be replaced by when the costs of NGS are reduced by an order of magnitude.

\section{Additional files}

Additional file 1: Confirmation of chromosomal aberrations detected by G-banding karyotype in 32 children with ID/DD, ASD and MCA. (XLSX 12 kb)

Additional file 2: Recurrent pathogenic/likely pathogenic CNVs detected by array-CGH in 36 children with ID/DD, ASD and MCA. (XLSX $14 \mathrm{~kb})$

Additional file 3: Non-recurrent pathogenic/likely pathogenic CNVs detected by array-CGH in 26 children with ID/DD, ASD and MCA. (XLSX $14 \mathrm{~kb}$ )

Additional file 4: Children's patients with two pathogenic/likely pathogenic CNVs. (XLSX $11 \mathrm{~kb}$ )

Additional file 5: CNVs of uncertain significance detected by array-CGH in 15 children with ID/DD, ASD and MCA (List 1), cnnLOH detected by
$\mathrm{CGH}+$ SNP microarrays in 8 children with ID/DD, ASD and MCA (List 2). (XLSX $17 \mathrm{~kb}$ )

Additional file 6: Likely benign CNVs detected by array-CGH in 50 children with ID/DD, ASD and MCA. (XLSX $14 \mathrm{~kb}$ )

\section{Abbreviations}

array-CGH: array-based comparative genomic hybridization; ASD: Autism spectrum disorders; cnnLOH: copy-number neutral loss of heterozygosity; CNV: Copy number variant; CNVs: Copy number variants; FISH: Fluorescence in situ hybridization; FoSTeS: Fork stalling and template switching; ID/ DD: Intellectual disability and developmental delay; MCA: Multiple congenital anomalies; MLPA: Multiplex ligation-dependent probe amplification; NAHR: Non-allelic homologous recombination; NGS: "Next-generation" sequencing; NHEJ: Non-homologous end joining; qPCR: quantitative polymerase chain reaction; VOUS: Variant of uncertain significance; WES: Whole-exome sequencing; WGS: Whole-genome sequencing

\section{Acknowledgments}

We would like to thank all the physicians and medical staff providing the specialized medical examinations supporting and completing the diagnosis of our patients. We would also like to thank patients and their parents for their participation in our study.

\section{Authors' contributions}

MW, JS, W and EH analysed and interpreted the array-CGH data regarding the phenotype. MW, HF, W and EH performed the confirmatory analyses using FISH, MLPA and relative $\mathrm{PPCR}$. MH, MV, BG and PN provided the cytogenetic analyses of karyotypes using G-banding. RG as a head physician coordinated the clinical genetics counselling in patients and their families. PK as a head of cytogenetics and molecular cytogenetics laboratories coordinated the laboratory part of the research. MW was a major contributor in writing the manuscript. All authors read and approved the final manuscript.

\section{Funding}

This work vas Supported by the project of Faculty of Science, Masaryk University, Brno, Czech republic MUNI/A/0958/2018. The institution has provided laboratory equipment and reagents required for analyses and financial support to obtain scientific data. The Department of Medical Genetics (University Hospital Brno) has provided the collection of biological material (DNA samples) and laboratory reagents and equipment to obtain scientific data, the physicians have carried out a specialized genetic counselling arising from the interpretation of scientific data. The researches from both involved institutions contributed equally in obtaining scientific data and their interpretation.

\section{Availability of data and materials}

Microarray data are available in the ArrayExpress database (www.ebi.ac.uk/ arrayexpress) under accession numbers E-MTAB-6685, E-MTAB-6733, E-MTAB6731, E-MTAB-6734, E-MTAB-6735 [47]. The other supporting data, i.e. data from cytogenetic analyses of karyotype (G-banding) and confirmatory analyses (FISH, MLPA, relative $\mathrm{QPCR}$ ) are available from the corresponding author on the reasonable request.

\section{Ethics approval and consent to participate}

Written institutional informed consent (the University Hospital Brno, Czech Republic) was obtained from the parents of the patients before the procedure of any genetic analyses. The informed consent is in accordance with the general recommendations of the Society of Medical Genetics and Genomics (Czech Medical Society of JE Purkyne). The study and informed consent were approved by the Ethical Committee of the University Hospital Brno (reference number 01-110112). Our study does not include any personal information leading to the identification of patients or their families.

Consent for publication

Not applicable.

Competing interests

The authors declare that they have no competing interests. 


\section{Received: 5 September 2018 Accepted: 16 July 2019}

\section{Published online: 23 July 2019}

\section{References}

1. Tassé MJ, Luckasson R, Nygren M. AAIDD proposed recommendations for ICD-11 and the condition previously known as mental retardation. Intellect Dev Disabil. 2013;51(2):127-31.

2. Michelson DJ, Shevell MI, Sherr EH, Moeschler JB, Gropman AL, Ashwal S. Evidence report: genetic and metabolic testing on children with global developmental delay: report of the quality standards Subcommittee of the American Academy of neurology and the practice Committee of the Child Neurology Society. Neurology. 2011;77(17):1629-35.

3. Kaufmann L, Ayub M, Vincent JB. The genetic basis of non-syndromic intellectual disability: a review. J Neurodev Disord. 2010;2(4):182-209.

4. Karam SM, Riegel M, Segal SL, Félix TM, Barros AJ, Santos IS, et al. Genetic causes of intellectual disability in a birth cohort: a population-based study. Am J Med Genet. 2015;167(6):1204-44.

5. Iwase S, Bérubé NG, Zhou Z, Kasri NN, Battaglioli E, Scandaglia M, et al Epigenetic etiology of intellectual disability. J Neurosci. 2017;37(45):10773-82.

6. Weise A, Mrasek K, Klein E, Mulatinho M, Llerena JC Jr, Hardenkopf D, et al. Microdeletion and microduplication syndromes. J Histochem Cytochem. 2012;60(5):346-58

7. Watson CT, Marques-Bonet T, Sharp AJ, Mefford HC. The genetics of microdeletion and microduplication syndromes: an update. Annu Rev Genomics Hum Genet. 2014;15:215-44.

8. Hastings PJ, Lupski JR, Rosenberg SM, Ira G. Mechanisms of change in gene copy number. Nat Rev Genet. 2009;10(8):551-64.

9. Lee JA, Carvalho CM, Lupski JR. A DNA replication mechanism for generating nonrecurrent rearrangements associated with genomic disorders. Cell. 2007:131(7):1235-47.

10. Zhang F, Khajavi M, Connolly AM, Towne CF, Batish SD, Lupski JR. The DNA replication FOSTES/MMBIR mechanism can generate genomic, genic and exonic complex rearrangements in humans. Nat Genet. 2009;41(7):849-53.

11. Di Gregorio E, Savin E, Biamino E, Belligni EF, Naretto VG, D'Alessandro G, et al. Large cryptic genomic rearrangements with apparently normal karyotypes detected by array-CGH. Mol Cytogenet. 2014;7(1):82.

12. Miller DT, Adam MP, Aradhya S, Biesecker LG, Brothman AR, Carter NP, et al. Consensus statement: chromosomal microarray is a first-tier clinical diagnostic test for individuals with developmental disabilities or congenital abnormalities. Am J Hum Genet. 2010;86(5):749-64.

13. Battaglia A, Doccini V, Bernardini L, Novelli A, Loddo S, Capalbo A, et al. Confirmation of chromosomal microarray as a first-tier clinical diagnostic test for individuals with developmental delay, intellectual disability, autism spectrum disorders and dysmorphic features. Eur J Paediatr Neurol. 2013; 17(6):589-99.

14. de Leeuw N, Dijkhuizen T, Hehir-Kwa JY, Carter NP, Feuk L, Firth HV, et al. Diagnostic interpretation of array data using public databases and internet sources. Hum Mutat. 2012;33(6):930-40.

15. Nowakowska B. Clinical interpretation of copy number variants in the human genome. J Appl Genet. 2017:58(4):449-57.

16. Buysse K, Delle Chiaie B, Van Coster R, Loeys B, De Paepe A, Mortier G, et al. Challenges for CNV interpretation in clinical molecular karyotyping: lessons learned from a 1001 sample experience. Eur J Med Genet. 2009:52(5):398403.

17. Hills A, Ahn JW, Donaghue C, Thomas H, Mann K, Ogilvie CM. MLPA for confirmation of array $\mathrm{CGH}$ results and determination of inheritance. Mol Cytogenet. 2010;3:19.

18. Kang SH, Shaw C, Ou Z, Eng PA, Cooper ML, Pursley AN, et al. Insertional translocation detected using FISH confirmation of array-comparative genomic hybridization (aCGH) results. Am J Med Genet A. 2010;152A(5): $1111-26$.

19. Wester U, Bondeson ML, Edeby C, Anneren G. Clinical and molecular characterization of individuals with 18p deletion: a genotype-phenotype correlation. Am J Med Genet A. 2006;140(11):1164-71.

20. D'Arrigo S, Gavazzi F, Alfei E, Zuffardi O, Montomoli C, Corso B, et al. The diagnostic yield of array comparative genomic hybridization is high regardless of severity of intellectual disability/developmental delay in children. J Child Neurol. 2016:31(6):691-9.

21. Carreira IM, Ferreira SI, Matoso E, Pires LM, Ferrão J, Jardim A, et al. Copy number variants prioritization after array-CGH analysis - a cohort of 1000 patients. Mol Cytogenet. 2015;8:103.
22. Hochstenbach R, van Binsbergen E, Engelen J, Nieuwint A, Polstra A, Poddighe $P$, et al. Array analysis and karyotyping: workflow consequences based on a retrospective study of 36,325 patients with idiopathic developmental delay in the Netherlands. Eur J Med Genet. 2009;52(4):161-9.

23. Sansović I, Ivankov AM, Bobinec A, Kero M, Barišić I. Chromosomal microarrays in clinical diagnosis: a study of 337 patients with congenital anomalies and developmental delays or intellectual disability. Croat Med J. 2017:58(3):231-8.

24. De Vries BB, Pfundt $R$, Leisink $M$, Koolen DA, Vissers LE, Janssen IM, et al. Diagnostic genome profiling in mental retardation. Am J Hum Genet. 2005; 77(4):606-16.

25. Veltman JA, Brunner HG. Understanding variable expressivity in microdeletion syndromes. Nat Genet. 2010;42(3):192-3.

26. Rosenfeld JA, Coe BP, Eichler EE, Cuckle H, Shaffer LG. Estimates of penetrance for recurrent pathogenic copy-number variations. Genet Med. 2013;15(6):478-81

27. Chen $C P$, Lin $S P$, Lee $C L$, Chern $S R, W u P S$, Chen $Y N$, et al. Familial transmission of recurrent 15q11.2 (BP1-BP2) microdeletion encompassing NIPA1, NIPA2, CYFIP1, and TUBGCP5 associated with phenotypic variability in developmental, speech, and motor delay. Taiwan J Obstet Gynecol. 2017; 56(1):93-7.

28. Girirajan S, Rosenfeld JA, Cooper GM, Antonacci F, Siswara P, Itsara A, et al. A recurrent 16p12.1 microdeletion supports a two-hit model for severe developmental delay. Nat Genet. 2010;42(3):203-9.

29. Wilfert AB, Sulovaru A, Turner TN, Coe BP, Eichler EE. Recurrent de novo mutations in neurodevelopmental disorders: properties and clinical implications. Genome Med. 2017;9(1):101.

30. Depaepe V, Suarez-Gonzales N, Dufour A, Passante L, Gorski JA, Jones KR, et al. Ephrin signalling controls brain size by regulation apoptosis of neural progenitors. Nature. 2005;435(7046):1244-50.

31. Traylor RN, Fan Z, Hudson B, Rosenfeld JA, Shaffer LG, Torchia BS, et al. Microdeletion of 6q16.1 encompassing EPHA7 in a child with mild neurological abnormalities and dysmorphic features: case report. Mol Cytogenet. 2009;2:17.

32. Rice AM, McLysaght A. Dosage sensitivity is a major determinant of human copy number variant pathogenicity. Nat Commun. 2017;8:14366.

33. Conrad B, Antonarakis SE. Gene duplication: a drive for phenotypic diversity and cause of human disease. Annu Rev Genomics Hum Genet. 2007:8: $17-35$.

34. Stankiewicz P, Pursley AN, Cheung SW. Challenges in clinical interpretation of microduplications detected by array CGH analysis. Am J Med Genet. 2010;152A(5):1089-100.

35. Fan YS, Quyang X, Peng J, Sacharow S, Tekin M, Barbouth D, et al. Frequent detection of parental consanguinity in children with developmental disorders by a combined CGH and SNP microarray. Mol Cytogenet. 2013; 6(1):38.

36. Wiszniewska J, Bi W, Shaw C, Stankiewicz P, Kang SH, Pursley AN, et al. Combined array CGH plus SNP genome analysis in a single assay for optimized clinical testing. Eur J Hum Genet. 2014;22(1):79-87.

37. Woodley MA, Bell E. Consanguinity as a major predictor of levels of democracy: a study of 70 nations. J Cross Cult Psychol. 2012:44(2):263-80.

38. Giorgio E, Ciolfi A, Biamino E, Caputo V, Di Gregorio E, Belligni EF, et al. Whole exome sequencing is necessary to clarify ID/DD cases with de novo copy number variants of uncertain significance: two proof-of-concept examples. Am J Hum Genet A. 2016;170(7):1772-9.

39. Erzurumluoglu AM, Shihab HA, Rodriguez S, Gaunt TR, Day IN. Importance of genetic studies in consanguineous populations for the characterization of novel human gene functions. Ann Hum Genet. 2016;80(3):187-96.

40. Silva M, de Leeuw N, Mann K, Schuring-Blom H, Morgan S, Giardino D, et al. European guidelines for constitutional cytogenomic analysis. Eur J Hum Genet. 2019;27(1):1-16.

41. Vermeesch JR, Brady PD, Sanlaville D, Kok K, Hastings RJ. Genome-wide arrays: quality criteria and platforms to be used in routine diagnostics. Hum Mutat. 2012:33(6):906-15.

42. Manning M. Hudgins L; professional practice and guidelines committee. Array-based technology and recommendation for utilization in medical genetics practice for detection of chromosomal abnormalities. Genet Med. 2010;12(11):742-5.

43. Liehr T, Schreyer I, Kuechler A, Manolakos E, Singer S, Dufke A, et al. Parental origin of deletions and duplications - about the necessity to check for cryptic inversions. Mol Cytogenet. 2018;11:20. 
44. Kirchhoff M, Bisgaard AM, Bryndorf T, Gerdes T. MLPA analysis for a panel of syndromes with mental retardation reveals imbalances in $5.8 \%$ of patients with mental retardation and dysmorphic features, including duplications of the Sotos syndrome and Williams-Beuren syndrome regions. Eur J Med Genet. 2007;50(1):33-42.

45. Hehir-Kwa JY, Pfundt R, Veltman JA. Exome sequencing and whole genome sequencing for the detection of copy number variation. Expert Rev Mol Diagn. 2015;15(8):1023-32.

46. Wayhelova M, Oppelt J, Smetana J, Hladilkova E, Filkova H, Makaturova E, et al. Novel de novo frameshift variant in the ASXL3 gene in a child with microcephaly and global developmental delay. Mol Med Rep. 2019;20(1): $505-12$.

47. Kolesnikov N, Hastings E, Keays M, Melnichuk O, Tang YA, Williams E, et al. ArrayExpress update--simplifying data submissions. Nucleic Acids Res. 2015; 43(D1):D1113-6.

\section{Publisher's Note}

Springer Nature remains neutral with regard to jurisdictional claims in published maps and institutional affiliations.

Ready to submit your research? Choose BMC and benefit from:

- fast, convenient online submission

- thorough peer review by experienced researchers in your field

- rapid publication on acceptance

- support for research data, including large and complex data types

- gold Open Access which fosters wider collaboration and increased citations

- maximum visibility for your research: over $100 \mathrm{M}$ website views per year

At BMC, research is always in progress.

Learn more biomedcentral.com/submissions 